\title{
Effect of Prewhirl on the Performance of Centrifugal Compressors
}

\author{
Yousef S. H. Najjar \\ Mechanical Engineering Department, Jordan University of Science \& Technology, Irbid, Jordan \\ Saleh A. M. S. Akeel \\ Mechanical Engineering Department, Umm Al-Qura University, Mekkah, Saudi Arabia
}

In comparison with axial flow compressors, centrifugal compressors have distinct relative merits such as: higher stage pressure ratio, simplicity and ruggedness of construction, shorter length, better resistance to foreign object damage, less vulnerable to blockage, handling small volume flows, better matching characteristics, and less manufacturing costs. However, the axial flow compressor has a large flow capture per unit area which is important to aircraft applications. In this study, prewhirl is introduced to alleviate the compressibility effect at the convex side of the eye to avoid the formation of shock waves and the consequent losses. However, this caused reduction of work absorbing capacity $W$ and pressure ratio $R$. This problem was overcome by twisting the prewhirl vanes between the tip and the root linearly, then parabolically, where the prewhirl angle $\alpha_{w 1}$ is maximum at tip $\alpha_{w 1 t}$ and zero at $\operatorname{root} \alpha_{w 1 r} \cdot \alpha_{w 1 t}$ was varied over a range of $20^{\circ}-50^{\circ}$. Computations showed that the linear variation recovered about $40 \%$ of $W$ and $R$, whereas the parabolic variation of $\alpha_{w 1 t}$ up to $40^{\circ}$ recovered $W$ and $R$ totally.

Keywords Centrifugal compressors; Performance; Prewhirl

The object of a good compressor design is to obtain the most air through a given diameter compressor, with a minimum number of stages while maintaining high efficiencies and aerodynamic stability over the operating range (Bathie, 1996). A centrifugal compressor achieves part of the compression process by causing the fluid to move outward in the centrifugal force field

Received 7 July 2000; accepted 12 July 2001.

Address correspondence to Professor Yousef S. H. Najjar, Mechanical Engineering Department, Jordan University of Science and Technology, Box 3030, Irbid 22110, Jordan. E-mail: y_najjar@ hotmail.com produced by the rotation of the impeller. Instead of arising from the conversion of kinetic energy into thermal energy in divergent passages, as in the rotor of the axial flow compressor, it arises from the change in potential energy of the fluid in the centrifugal force field of the impeller. It is therefore, less limited by the problems of boundary-layer growth and separation in adverse pressure gradient (Kerrebrock, 1992).

Due to lower mass capture per unit frontal area, centrifugal compressors are widely used in small gas turbine engines such as small turboprops, turboshafts and auxiliary power units (APUs), and for the high-pressure spools in small turbofans.

Centrifugal compressors in comparison with axial flow compressors have some relative merits, such as higher stage pressure ratio, simplicity and ruggedness of construction, suitability to handle small volume flows, shorter length, better resistance to foreign object damage (FOD), less vulnerability to blockage and the ability to operate efficiently over a wide range of mass flow between surging and choking at a particular rotational speed (alleviating the problem of matching of operating conditions with those of the associated components) and flow direction of discharge air that is convenient for the installation of an intercooler and/or heat exchanger, Kerrebrock (1992) and Cohen, Rogers, and Saravanamuttoo (1998). Centrifugal compressors are used when the size is small enough for the Reynolds number of the flow to become small, and when the blade tip clearance becomes relatively large. They will cost less to manufacture than the equivalent multistage axial compressor in almost every size, Wilson and Korakianitis (1999).

Generally, dynamic compressors convert velocity to pressure continuously. This is in comparison with the rotary compressor of the vane or screw or other type, which compresses continuously in portions separated by moving walls and boundaries, Harman (1981).

Assuming the air flow approaches the compressor intake axially, then before passing into the radial channels of the impeller, the air flow is deflected through a certain angle in the eye of 


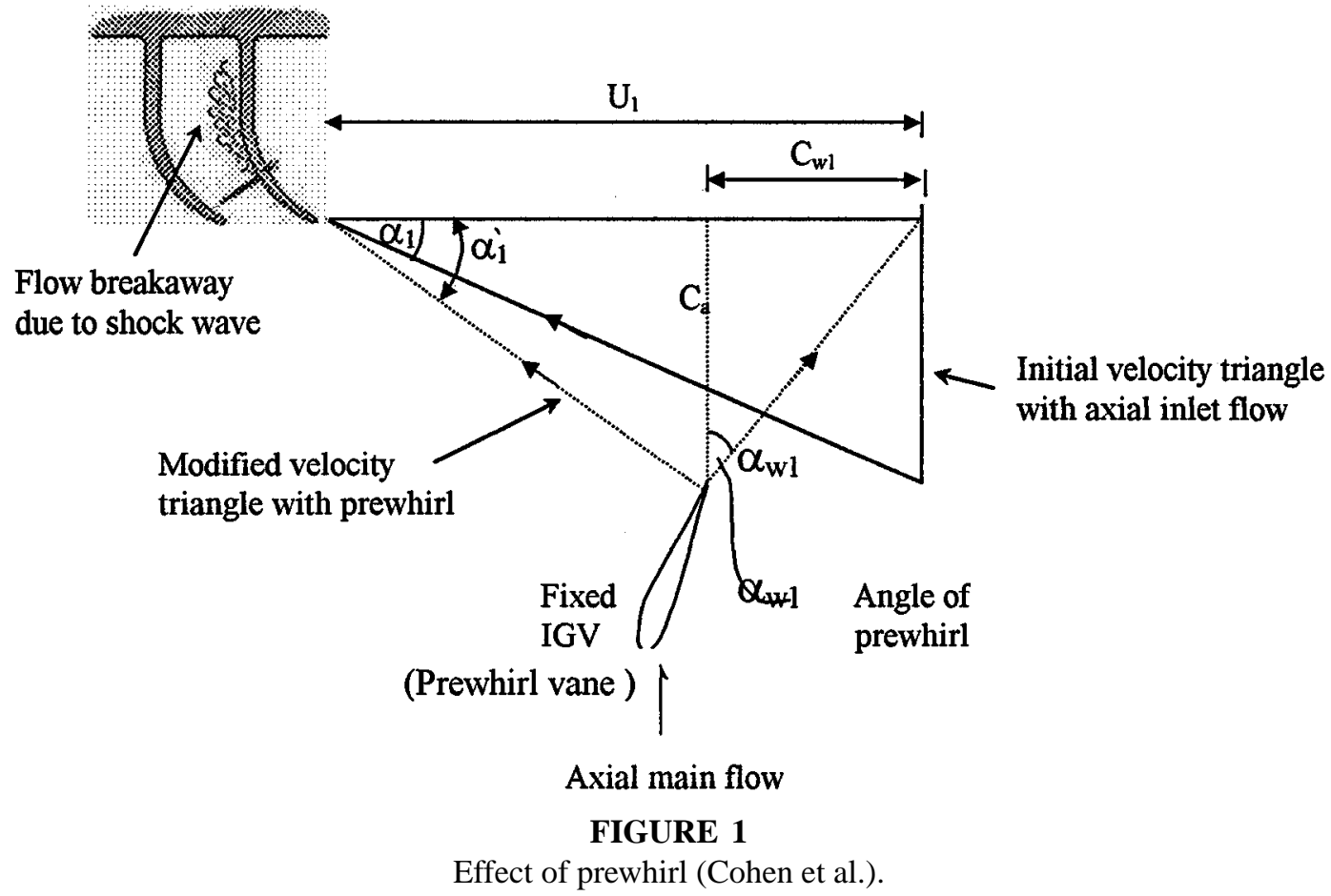

the impeller where there is a possibility for the flow to break away from the convex face, which may lead to a shock wave with consequent losses. In this case the velocity relative to the vane $V_{1}$ will be maximum at the eye tip where the vane speed $U$ is greater. Hence, the maximum Mach number is at the eye tip $M_{v 1 t}$ given by:

$$
M_{v 1 t}=V_{1 t} / \sqrt{\gamma R_{0} T_{1}}
$$

where $T_{1}$ is the static temperature at the inlet, and it is the lowest in the flow passage. Taking $C_{w 2}$ as the whirl component at impeller tip $r_{2}$ then the torque, which is equivalent to the change of angular momentum, is given by: torque $=C_{w 2} r_{2}$, and Power $=C_{w 2} r_{2} \omega=C_{w 2} U_{2}$. Hence introducing the slip factor $\sigma=C_{w 2} / U_{2}$ and the power input factor $\psi$, which takes into account friction between the casing and the air carried round by the vanes, plus disc friction or windage, results in actual power $=$ $\psi \sigma U_{2}^{2}$.

Although $M_{v 1 t}$ may be satisfactory under ground conditions (about 0.9), it becomes excessively high at altitude if installed in an aircraft gas turbine engine (more than 1.0). Assuming that $T_{\text {alt }} / T_{g r} \sim \frac{3}{4}$, then $M_{v 1, \text { alt }} / M_{v 1, g r} \sim 1$.15. Flow distortions due to aircraft maneuvers may cause a more severe change in flows and hence $M$, thereby they may have a significant impact on compressor stability. However, our main concern in this work is to the effect of prewhirl on the compressor performance. Hence, it becomes necessary to reduce $V_{1}$ by introducing prewhirl at the intake, which involves passing the air over curved inlet guide vanes attached to the compressor casing, before being drawn into the impeller eye. Assuming the same mass flow in both cases, axial velocity $C_{a}$ remains nearly the same, however $V_{1}$ is reduced and the curvature of impeller vanes at inlet is reduced, i.e. $\alpha_{1}$ increases as shown in Figure 1.

\section{THEORETICAL ANALYSIS}

Since the air flow has an initial whirl velocity $C_{w 1}$ then, by introducing prewhirl using inlet guide vanes (IGV) as shown in Figure 1, the torque which equals $\left(C_{w 2} r_{2}-C_{w 1} r_{1}\right)$ is reduced. Assuming $C_{w 1}$ is constant over the eye, $C_{w 1} r_{1}$ will increase from root to tip. Hence, the work done on each unit mass flow of air depends on the radius at which it enters the eye. Since $M_{v 1}$ is high only at eye tip, it is preferable to vary the prewhirl angle $\alpha_{w 1}$ gradually reducing it from a maximum at the tip to zero at the root of the eye. Thereby, the prewhirl vanes get twisted, Cohen et al. (1998).

In this work, three patterns of twisting the IGV are considered.

\section{Case 1}

Constant $\alpha_{w 1}$ from root to tip. Hence $C_{w 1}=C_{a} \tan \alpha_{w 1}=$ constant value along the eye. But $C_{w 1} r_{1}$ increases from root to tip, thereby also increasing the drop in the work capacity from root to tip. For this case $\alpha_{w 1}$ was taken $30^{\circ}$. Hence

$$
\begin{aligned}
\text { Torque } & =\sigma U_{2} r_{2}-C_{w 1} r_{m} \\
\text { Work } & =\psi\left[\sigma U_{2}^{2}-C_{w 1} r_{m}\left(2 \pi r_{m}\right)\right]=C_{p} \Delta T_{0}
\end{aligned}
$$


TABLE I

Variation of performance parameters with prewhirl angle $\alpha_{w 1}$ (constant from root tip)

\begin{tabular}{cccccc}
\hline $\begin{array}{l}\text { Prewhirl } \\
\text { angle } \alpha_{w}\end{array}$ & $\begin{array}{c}\text { Torque } \\
(\mathrm{kJ})\end{array}$ & $\begin{array}{c}\text { Work done } \\
(\mathrm{kjJ})\end{array}$ & $\begin{array}{c}\Delta T_{0} \\
(\mathrm{~K})\end{array}$ & $R$ & $M_{v 1 t}$ \\
\hline $0^{\circ}$ & 102.5 & 186.76 & 193.26 & 4.24 & 0.91 \\
$20^{\circ}$ & 96.64 & 176.08 & 182.21 & 3.96 & 0.78 \\
$30^{\circ}$ & 93.21 & 169.829 & 175.74 & 3.80 & 0.70 \\
$40^{\circ}$ & 89 & 162.16 & 167.81 & 3.62 & 0.62 \\
$50^{\circ}$ & 83.33 & 151.82 & 157.11 & 3.374 & 0.52 \\
\hline
\end{tabular}

These performance parameters are calculated for different values of $\alpha_{w 1 t}$, namely $20^{\circ}, 30^{\circ}, 40^{\circ}, 50^{\circ}$. The results are shown in Table I compared with the case of no prewhirl.

\section{Case 2}

$\alpha_{w 1}$ increases linearly from root to tip of the eye. It starts with $\alpha_{w 1}=0$ at root. Hence,

$$
\begin{aligned}
& C_{w 1}=C_{a} \tan \alpha_{w 1} \\
& \left(\frac{C_{w 1}}{\tan \alpha_{w 1}}\right)=\text { Const }=\left(\frac{C_{w 1}}{\tan \alpha_{w 1}}\right)_{r}=\left(\frac{C_{w 1}}{\tan \alpha_{w 1}}\right)_{t}=\ldots
\end{aligned}
$$

Take $\left(r / r_{t}\right)=r_{e}$

$$
\tan \alpha_{w 1}=a r_{e}+b
$$

where " $a$ " and " $b$ " are constants which can be found by choosing certain values for $\mathrm{r}_{r} / r_{t}$ and $\alpha_{w 1}$ then, solving two equations by the boundary conditions at root and tip. Hence for $r_{r} / r_{t}=0.54$, and $\alpha_{w 1 t}=20^{\circ}$ the linear variation of $\alpha_{w 1}$ along the eye can be given by:

$$
\begin{aligned}
\tan \alpha_{w 1} & =0.728 r_{e}-0.364 \\
C_{w 1} & =C_{a} \tan \alpha_{w 1}
\end{aligned}
$$

Thereby,

$$
\left(C_{w 1} r\right)_{m}=\int_{r r}^{r t} C_{a}\left[0.728\left(r / r_{t}\right)-0.364\right] r_{t} d\left(r / r_{t}\right)
$$

TABLE II

Variation of performance parameters with prewhirl angle $\alpha_{w 1}$ (linear variation from root to tip)

\begin{tabular}{cccccc}
\hline $\begin{array}{l}\text { Prewhirl } \\
\text { angle } \alpha_{w}\end{array}$ & $\begin{array}{c}\text { Torque } \\
(\mathrm{kJ})\end{array}$ & $\begin{array}{c}\text { Work done } \\
(\mathrm{kJ})\end{array}$ & $\begin{array}{c}\Delta T_{0} \\
(\mathrm{~K})\end{array}$ & $R$ & $M_{v 1 t}$ \\
\hline $0^{\circ}$ & 102.5 & 168.76 & 193.26 & 4.24 & 0.91 \\
$20^{\circ}$ & 100.55 & 183.2 & 198.58 & 4.15 & 0.78 \\
$30^{\circ}$ & 99.4 & 181.12 & 187.42 & 4.09 & 0.70 \\
$40^{\circ}$ & 98 & 178.56 & 184.78 & 4.02 & 0.62 \\
$50^{\circ}$ & 96.11 & 175.11 & 181.21 & 3.94 & 0.52 \\
\hline
\end{tabular}

After some mathematical manipulations, one can obtain

$$
\left(C_{w 1} r\right)_{m}=0.091 C_{a} r_{t}
$$

Alternatively, carrying out numerical integration using the trapezoidal method, the result in Equation (13) is obtained. This procedure is repeated for the other values of $\alpha_{w 1 t}$, namely $30^{\circ}, 40^{\circ}$ and $50^{\circ}$. The results are shown in Table II in comparison with the case of no prewhirl.

\section{Case 3}

$\alpha_{w 1}$ increases in a parabolic relation from $0^{\circ}$ at root to $\alpha_{w 1 t}$ at tip.

$$
\begin{aligned}
& C_{w 1}^{2}=C_{a} \tan \alpha_{w 1} \\
& \left(\frac{C_{w 1}^{2}}{\tan \alpha_{w 1}}\right)=\text { const. }=\left(\frac{C_{w 1}^{2}}{\tan \alpha}\right)_{r}=\left(\frac{C_{w 1}^{2}}{\tan \alpha_{1}}\right)_{t}=\ldots
\end{aligned}
$$

$$
C_{w 1}=\sqrt{C_{a}} \tan \alpha_{w 1}
$$

Take $\tan \alpha_{w 1}=a r_{e}+b$

Then using $r_{e}=0.5$ and $\alpha_{w 1 t}=20^{\circ}$, the result is an equation similar to that produced previously namely,

$$
\tan \alpha_{w 1}=0.728 r_{e}-0.364
$$

Consequently,

$$
C_{w 1}=\sqrt{C_{a}\left[0.364\left\{2\left(r / r_{t}\right)\right\}-1\right]}
$$

After some mathematical manipulations and where $C_{a}=$ $143 \mathrm{~m} / \mathrm{s}$ is taken to provide a suitable compromise between high flow per unit frontal area and low frictional losses in the intake,

$$
C_{w 1}=7.215\left[2\left(r / r_{t}\right)-1\right]^{1 / 2}
$$




\section{TABLE III}

Variation of performance parameters with prewhirl angle $\alpha_{w 1}$ (parabolic variation from root to tip)

\begin{tabular}{lcclcc}
\hline $\begin{array}{c}\text { Prewhirl } \\
\text { angle } \alpha_{w}\end{array}$ & $\begin{array}{c}\text { Torque } \\
(\mathrm{kJ})\end{array}$ & $\begin{array}{c}\text { Work done } \\
(\mathrm{kJ})\end{array}$ & $\begin{array}{c}\Delta T_{0} \\
(\mathrm{~K})\end{array}$ & \multicolumn{1}{c}{$R$} & $M_{v 1 t}$ \\
\hline $0^{\circ}$ & 102.5 & 186.76 & 193.26 & 4.24 & 0.91 \\
$20^{\circ}$ & 102.14 & 186.1 & 192.58 & 4.22 & 0.78 \\
$30^{\circ}$ & 102.05 & 185.93 & 192.4 & 4.22 & 0.70 \\
$40^{\circ}$ & 101.95 & 185.76 & 192.2 & 4.21 & 0.62 \\
$50^{\circ}$ & 101.85 & 185.57 & 192 & 4.21 & 0.52 \\
\hline
\end{tabular}

Then

$$
\begin{aligned}
\left(C_{w 1} r\right)_{m} & =\int_{r r}^{r t} 7.215\left[2\left(r / r_{t}\right)-1\right]^{1 / 2} r_{t} d\left(r / r_{t}\right) \\
& =1.082 \int_{0.5}^{1}\left[2\left(r / r_{t}\right)-1\right]^{1 / 2} d\left(r / r_{t}\right)
\end{aligned}
$$

After some manipulations, one can obtain

$$
\left(C_{w 1} r\right)_{m}=1.082 / 3=0.361
$$

Alternatively, by numerical integration of the relationship between $\mathrm{C}_{w 1}$ and $\sqrt{\tan \alpha_{w 1}}$, the result in Equation (22) is obtained. This procedure is repeated for the other values of $\alpha_{w 1 t}$, namely $30^{\circ}, 40^{\circ}$ and $50^{\circ}$. The results are shown in Table III compared with the case of no prewhirl.

\section{DISCUSSION OF RESULTS}

The particulars of geometry and the design point operating variables were chosen for a typical centrifugal compressor of a small aircraft engine, Cohen, Rogers, and Saravanamuttoo (1998) and Mattingly (1996). However the results are generalized for different geometries and operating variables.

These particulars are: impeller radius $r_{2}=0.25 \mathrm{~m}$; eye tip $r_{t}=0.15 \mathrm{~m}$; eye root radius $r_{r}=0.075 \mathrm{~m}$; rotational speed

TABLE IV

Variation of normalized performance parameters with prewhirl $\alpha_{w 1 t}$ (parabolic variation from root to tip)

\begin{tabular}{ccccc}
\hline $\begin{array}{l}\text { Prewhirl } \\
\text { Angle } \\
\alpha_{w}\end{array}$ & $\begin{array}{c}\text { Normalized } \\
\text { torque }\end{array}$ & $\begin{array}{c}\text { Normalized } \\
\text { work }\end{array}$ & $\begin{array}{c}\text { Normalized } \\
\text { pressure } \\
\text { ratio }\end{array}$ & $\begin{array}{c}\text { Normalized } \\
\text { mach number } \\
\text { at tip } M_{v 1 t}\end{array}$ \\
\hline $0^{\circ}$ & 1.00 & 1.00 & 1.00 & 1.00 \\
$20^{\circ}$ & 0.9665 & 0.9965 & 0.9953 & 0.857 \\
$30^{\circ}$ & 0.9956 & 0.9956 & 0.9953 & 0.769 \\
$40^{\circ}$ & 0.9946 & 0.9946 & 0.9929 & 0.681 \\
$50^{\circ}$ & 0.9937 & 0.9937 & 0.9929 & 0.571 \\
\hline
\end{tabular}

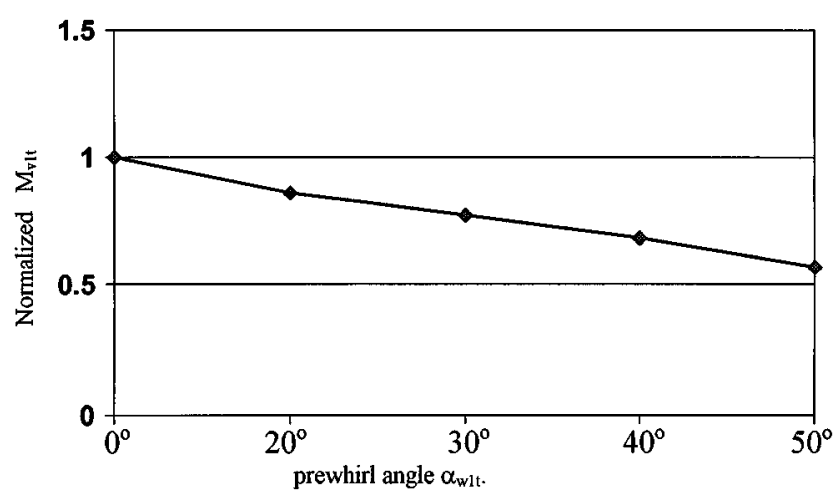

FIGURE 2

Variation of the normalized relative Mach No. $\left(M_{v 1 t}\right)$ with the prewhirl angle at eye tip $\alpha_{w 1 t}$.

$N=290 \mathrm{rps}$; air mass flow rate $m=9 \mathrm{~kg} / \mathrm{s}$; axial flow velocity $C_{a}=143 \mathrm{~m} / \mathrm{s}$; slip factor $\sigma=0.9$; power input factor $\psi=1.04$; compressor isentropic efficiency $\eta_{c}=0.78$; ambient conditions: $T_{01}=295 \mathrm{~K}$ and $P_{01}=1.1$ bars.

The main performance parameters namely: $M_{v 1}$, torque, work, and pressure ratio $R$ were calculated for the basic case of no prewhirl; and the three specified cases of prewhirl over a wide range of angles of $20^{\circ}-50^{\circ}$. The results are shown in Tables I-III. However, to generalize the results, they have been nondimensionalized by dividing the value of each performance parameter in these tables over its corresponding value at the basic case of no prewhirl. The results are shown in Table IV for the parabolic case for illustration, and all presented in Figures 2-4.

Figure 2 shows the variation of the normalized relative Mach. Number $M_{v 1 t}$, with $\alpha_{w 1 t}$. It is clear that $M_{v 1}$ can be decreased to $55 \%$ of its value at no prewhirl. This makes the operating conditions very safe as far as compressibility effect is concerned even at high altitude with aircraft engines. Figure 3 shows the variation of nondimensional work with $\alpha_{w 1 t}$ for the three cases

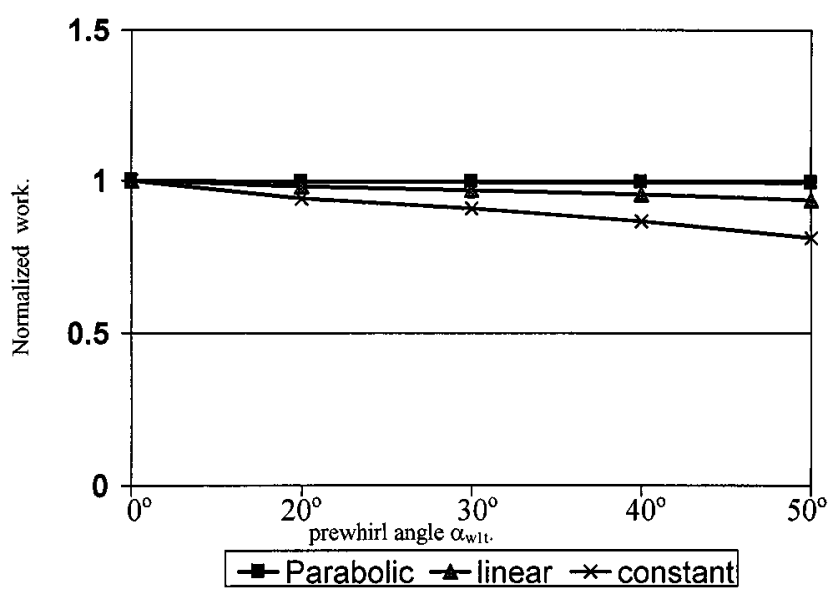

FIGURE 3

Variation of normalized work with prewhirl angle $\alpha_{w 1 t}$ for the three cases. 


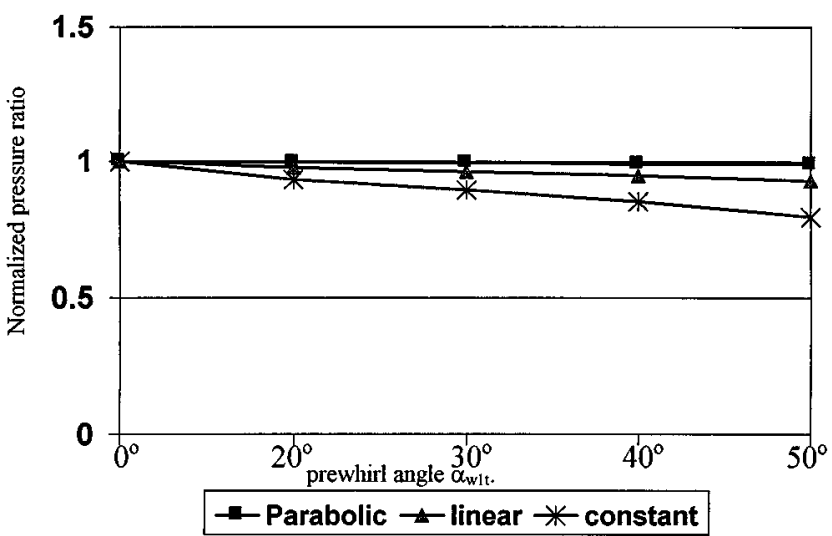

FIGURE 4

Variation of normalized pressure ratio with prewhirl angle $\alpha_{w 1 t}$ for the three cases.

of prewhirl. It is noticed that a sharp drop in the work absorbing capacity of the compressor takes place with constant angle of prewhirl, reduced to about half that value with linear variation of prewhirl. The parabolic variation shows nearly perfect recovery of work up to $\alpha_{w 1 t} \sim 40^{\circ}$. Similar trends are shown in Figure 4 for the obtainable pressure ratio.

\section{CONCLUSIONS}

In spite of alleviating the compressibility effect, introducing constant prewhirl along the height of the eye in centrifugal compressors reduces the work absorbing capacity $(W)$ and pressure ratio $(R)$ down to about $80 \%$.

Reducing the prewhirl angle, from tip to root of the eye linearly improves performance and increases $W$ and $R$, up to more than $94 \%$ of the nonprewhirl values.

Reduction of prewhirl angle in a parabolic relationship, restores $\mathrm{W}$ and $\mathrm{R}$ to the level of no prewhirl when using a prewhirl angle at tip up to $\alpha_{w 1 t}=40^{\circ}$. This is in addition to reducing the danger of compressibility effect.

\section{NOMENCLATURE}

$C_{a} \quad$ axial component of velocity $[\mathrm{m} / \mathrm{s}]$

$C_{p} \quad$ specific heat at constant pressure $[\mathrm{kJ} / \mathrm{kg} \mathrm{K}]$

$C_{w} \quad$ whirl component of absolute velocity $[\mathrm{m} / \mathrm{s}]$

IGV inlet guide vanes (prewhirl vanes)

$m$ air mass flow rate $[\mathrm{kg} / \mathrm{s}]$

$M \quad$ Mach number
$N \quad$ rotational speed [rps]

$P_{o} \quad$ stagnation pressure [bar]

$r_{e} \quad$ radial position between eye root and tip $\left[r / r_{t}\right]$

$r_{r} / r_{t}$ root to tip radius ratio of the eye

$r_{m} \quad$ mean radius of the eye [m]

$R \quad$ pressure ratio

$R_{O} \quad$ gas constant $[\mathrm{kJ} / \mathrm{kg} \mathrm{K}]$

$T_{o} \quad$ stagnation temperature $[\mathrm{K}]$

$T_{\text {alt }} \quad$ ambient temperature at altitude [K]

$T_{g r}$ ambient temperature at ground,

$U \quad$ impeller speed $[\mathrm{m} / \mathrm{s}]$

$V \quad$ relative velocity $[\mathrm{m} / \mathrm{s}]$

$W \quad$ work absorbing capacity [kJ]

\section{Greek Symbols}

$\alpha_{1} \quad$ vane angle [deg]

$\alpha_{1}^{\prime} \quad$ vane angle with prewhirl [deg]

$\alpha_{w} \quad$ prewhirl angle [deg]

$\sigma \quad$ slip factor

$\psi \quad$ power factor

$\omega \quad$ angular velocity [ $\mathrm{rad} / \mathrm{s}]$

$\eta_{c} \quad$ compressor efficiency

$\gamma \quad$ specific heat ratio for exit

\section{Subscripts}

1,2 stations at impeller inlet and exit

a axial component

$m$ mean radius of the eye

$r$ root radius of the eye

$t \quad$ tip radius of the eye

$w \quad$ whirl component

\section{REFERENCES}

Bathie, W. W. 1996. Fundamentals of gas turbines. 2nd ed. New York: John Wiley \& Sons.

Cohen, H., Rogers, G. F. C., and Saravanamuttoo, H. I. H. (1998) Gas turbine theory. 4th. ed. : Longman.

Harman, R. T. C. 1981. Gas turbine engineering. London: MacMillan Press.

Kerrebrock, J. L. 1992. Aircraft engines and gas turbines. 2nd ed. Cambridge, MA: MIT Press.

Mattingly, J. D. 1996. Elements of gas turbine propulsion. New York: McGraw-Hill.

Wilson, D. G., and Korakianitis, T. 1999. The design of high-efficiency turbomachinery and gas turbines. 2nd ed. Upper Saddle River, NJ: Prentice-Hall. 

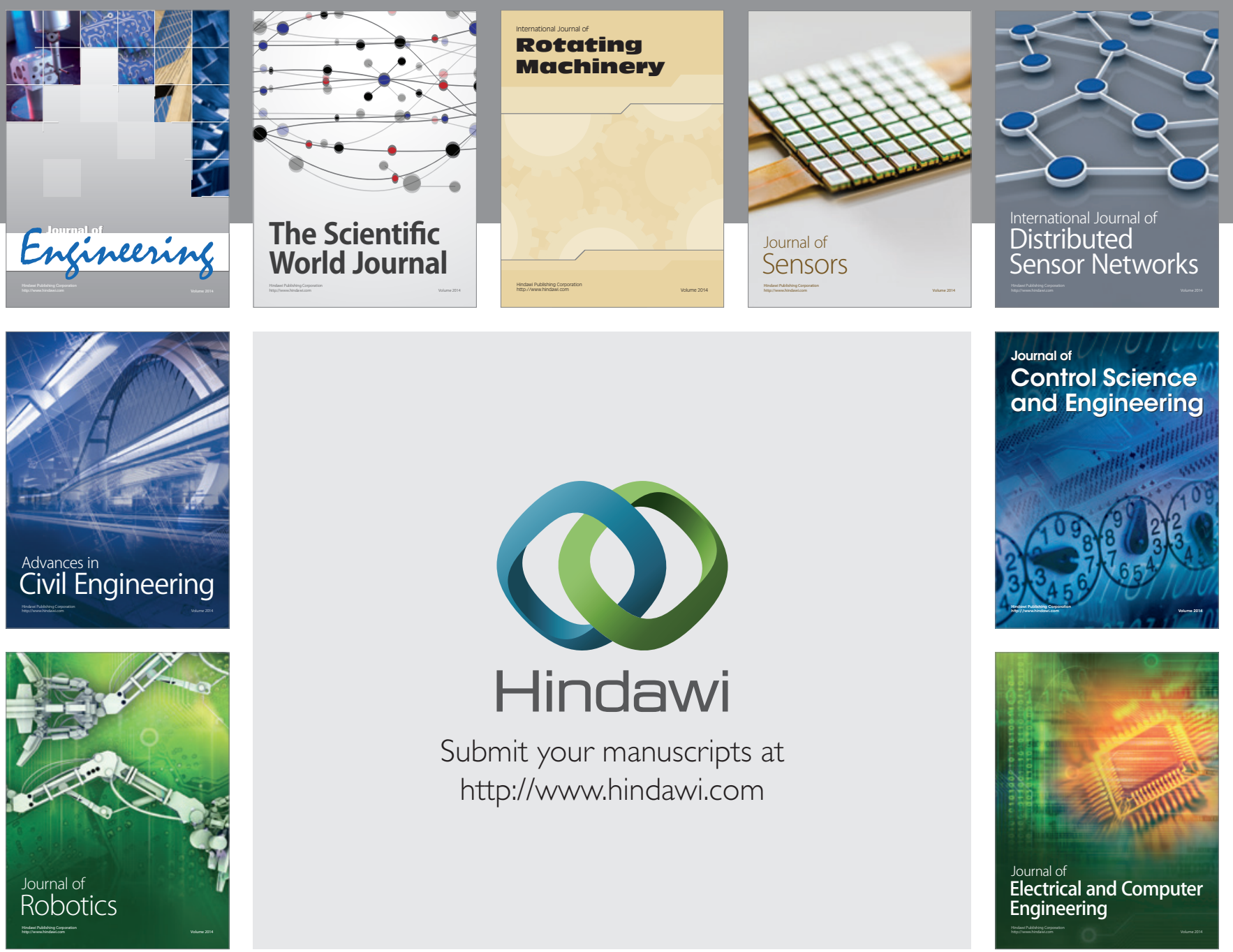

Submit your manuscripts at

http://www.hindawi.com
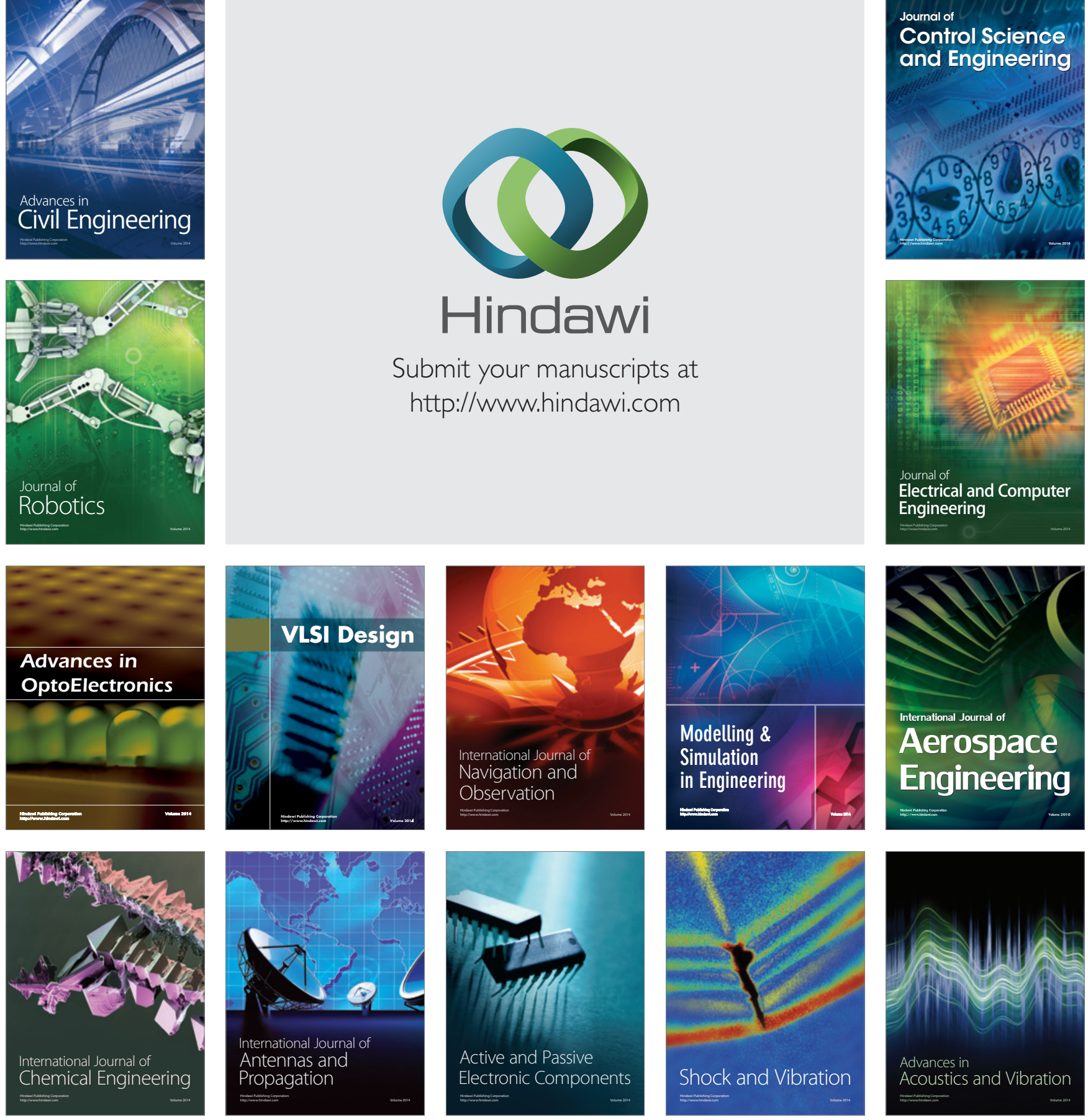